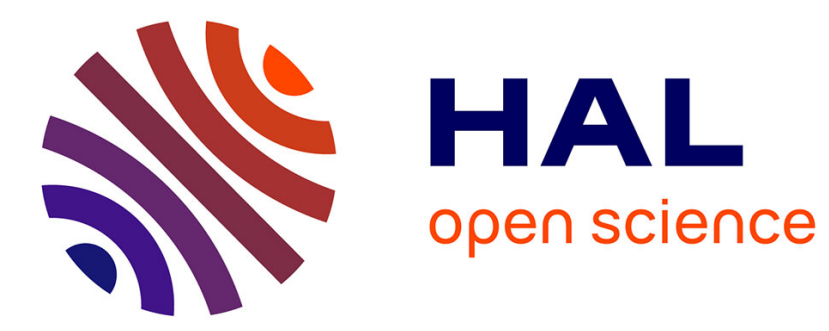

\title{
Un nouveau reçu de Syène/Éléphantine pour la capitation et la contribution des déserteurs
}

\author{
Jean-Luc Fournet
}

\section{To cite this version:}

Jean-Luc Fournet. Un nouveau reçu de Syène/Éléphantine pour la capitation et la contribution des déserteurs. Archiv für Papyrusforschung und verwandte Gebiete, 2014, 60 (1), pp.240-248. 10.1515/apf-2014-0120 . hal-01597540

\section{HAL Id: hal-01597540 \\ https://hal.science/hal-01597540}

Submitted on 28 Sep 2017

HAL is a multi-disciplinary open access archive for the deposit and dissemination of scientific research documents, whether they are published or not. The documents may come from teaching and research institutions in France or abroad, or from public or private research centers.
L'archive ouverte pluridisciplinaire HAL, est destinée au dépôt et à la diffusion de documents scientifiques de niveau recherche, publiés ou non, émanant des établissements d'enseignement et de recherche français ou étrangers, des laboratoires publics ou privés. 


\title{
Un nouveau reçu de Syène/Éléphantine pour la capitation et la contribution des déserteurs
}

\author{
Jean-Luc Fournet (Paris)
}

\begin{abstract}
Edition of a Roman receipt from Syene/Elephantine for the poll tax ( $\left.\lambda \alpha o \gamma \rho \alpha \varphi i^{\alpha}\right)$ and the contribution for those who have fled ( $\mu \varepsilon \rho \iota \sigma \mu o ̀ \varsigma ~ \varepsilon ं \pi \imath \varepsilon \varepsilon \varphi \alpha \lambda i ́ o v ~ \alpha ่ v \delta \rho \hat{\omega} v \dot{\alpha} v \alpha \kappa \varepsilon \chi \omega \rho \eta \kappa o ́ \tau \omega v)$.
\end{abstract}

Keywords: Syene/Elephantine, fiscality, poll tax, fiscal anachoresis, ostracon

Coll. Kauffmann (France) $)^{1}$

$\mathrm{H} 8,9 \times \mathrm{L} 7,7 \mathrm{~cm}$

Syène/Éléphantine 29/08-27/09/142

Cet ostracon, qui conserve un reçu fiscal émis pour la capitation et la contribution des déserteurs (cf. ci-dessous), entre dans un dossier de reçus émis par les fermiers Hérakleidês et Isidôros constitué, avec le présent document, de 20 pièces datées entre juillet/août 141 et le 6 novembre 143 (cf. Tableau 1).

Tableau 1: le dossier des reçus d'Hérakleidês et Isidôros

\begin{tabular}{|c|c|c|c|c|c|}
\hline & date & $\begin{array}{l}\text { année } \\
\text { régnale }\end{array}$ & assistant & taxe + année & montant \\
\hline 1- O.Wilck. 182 & $\begin{array}{l}27 / 07- \\
23 / 08 / \\
141\end{array}$ & $\delta$ & $\Pi \alpha[\chi o ́ \mu \psi \alpha \chi \chi 1 \varsigma]^{2}$ & $\begin{array}{l}\lambda \alpha о \gamma \rho \alpha \varphi^{\prime} \alpha \\
\delta L \\
\mu \varepsilon \rho . \dot{\varepsilon} \pi . \dot{\alpha} v \delta \rho . \\
\dot{\alpha} v \alpha \kappa . \beta L\end{array}$ & $\begin{array}{l}17 \mathrm{dr} .1 \mathrm{ob} . \\
{[---]}\end{array}$ \\
\hline 2- O.Wilck. 181 & $\begin{array}{l}3 / 08 / \\
141\end{array}$ & $\delta$ & 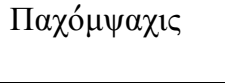 & 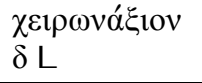 & 2 dr. 2 ob. \\
\hline 3-O.Wilck. 183 & $\begin{array}{l}1 / 10 / \\
141\end{array}$ & $\varepsilon$ & 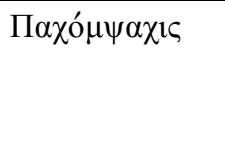 & 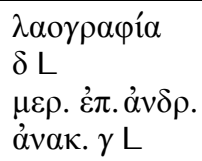 & $\begin{array}{l}17 \mathrm{dr} .1 \mathrm{ob} . \\
1 \mathrm{dr} .[+\mathrm{x} \\
\text { ob. }]^{3}\end{array}$ \\
\hline
\end{tabular}

\footnotetext{
${ }^{1}$ Je remercie M. Jean-Guy Kauffmann d'avoir mis à ma disposition cet ostracon de sa collection pour étude. Cette collection a été depuis cédée à l'«Association Bible \& Civilisations» (Jérusalem).

${ }^{2}$ L'image confirme bien que l'ostracon est de la main de Pakhompsakhis (et non de celle de Palakhêmis).

${ }^{3}$ Cf., ci-dessous, $1.12 \mathrm{n}$.
} 


\begin{tabular}{|c|c|c|c|c|c|}
\hline 4- O.Leid. 182 & $1 / 11 / 141$ & $\varepsilon$ & $\Sigma \alpha \rho \alpha \pi i ́ \omega v o \varsigma$ & $\begin{array}{l}\gamma \varepsilon \omega \mu \varepsilon \tau \rho i ́ \alpha \\
\varphi o t v \imath \kappa \hat{v} \vee{ }^{\prime} \\
{[\delta] \mathrm{L}}\end{array}$ & 26 dr. 4 ob. \\
\hline 5- P.Eleph.Wagner 30 & $5 / 12 / 141$ & $\varepsilon$ & 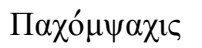 & $\mathrm{L}$ & 17 dr. 1 ob. \\
\hline 6- O.Bodl. II 966 & $141 / 142$ & $\varepsilon$ & $\Sigma \alpha \rho \alpha \pi i ́ \omega v o \varsigma$ & 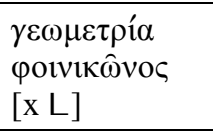 & $\begin{array}{l}6 \text { dr. rhyp. } \\
{[+\mathrm{x}]}\end{array}$ \\
\hline 7- O.Wilck. 189 & $\begin{array}{l}26 / 04- \\
24 / 06 / 142\end{array}$ & $\varepsilon$ & П $\alpha \chi o ́ \mu \psi \alpha \chi[1 \varsigma$ & $\begin{array}{l}\lambda \alpha o \gamma \rho \alpha \varphi^{\prime} \alpha \\
\varepsilon \mathrm{L}\end{array}$ & 17 dr. 1 ob. \\
\hline 8- P.Köln II 115 & $6 / 06 / 142$ & $\varepsilon$ & 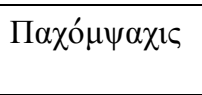 & 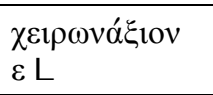 & 20 dr. 2 ob. \\
\hline 9- O.Wilck. 188 & $7 / 06 / 142$ & $\varepsilon$ & Пахó $\mu \psi \alpha \chi 1 \varsigma$ & $\begin{array}{l}\lambda \alpha o \gamma \rho \alpha \varphi^{\prime} \alpha \\
\delta \mathrm{L}\end{array}$ & 17 dr. $1 \mathrm{ob}$. \\
\hline 10- O.Bodl. II 967 & $26 / 06 / 142$ & $\varepsilon$ & П $\alpha \chi]$ ó $\mu \psi \alpha \chi 1 \varsigma$ &  & $\begin{array}{l}3 \text { dr. rhyp. } \\
2 \text { ob. }\end{array}$ \\
\hline 11- O.Kauffmann & $\begin{array}{l}29 / 08- \\
27 / 09 / 142\end{array}$ & s & П $\alpha \chi o ́ \mu[\psi \alpha \chi 1 \varsigma$ & $\begin{array}{l}\lambda \alpha o \gamma \rho \alpha \varphi^{\prime} \alpha \\
{\left[\varepsilon^{?}\right] \mathrm{L}} \\
\mu \varepsilon \rho . \dot{\varepsilon} \pi . \dot{\alpha} v \delta \rho . \\
\dot{\alpha} v \alpha \kappa . \gamma \mathrm{L}\end{array}$ & $\begin{array}{l}17 \text { dr. } 1 \text { ob. } \\
1 \text { dr. } 5 \text { ob. }\end{array}$ \\
\hline 12- O.Wilck. 190 & $6 / 09 / 142$ & 5 & 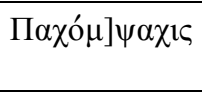 & $\begin{array}{l}\lambda \alpha o \gamma \rho \alpha \varphi^{\prime} \alpha \\
{[\varepsilon]\llcorner}\end{array}$ & 17 dr. 1 ob. \\
\hline 13-O.Wilck. 191 & $7 / 09 / 142$ & 5 & 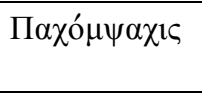 & $\begin{array}{l}\lambda \alpha o \gamma \rho \alpha \operatorname{li}^{\alpha} \alpha \\
\varepsilon \mathrm{L}\end{array}$ & 16 dr. $1 \mathrm{ob}$. \\
\hline 14- O.Bodl. II 1066 & $\begin{array}{l}19 / 04 / 143 \\
25 / 05 / 143\end{array}$ & $\varsigma$ & $\Pi \alpha \lambda \alpha ́ \chi \eta \mu 1 \varsigma^{4}$ & 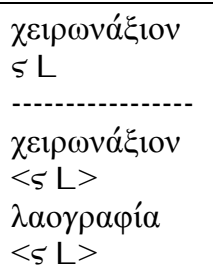 & $\begin{array}{l}8 \mathrm{dr} . \\
4 \text { dr. } \\
5 \mathrm{dr} .1 \text { ob. }\end{array}$ \\
\hline 15-O.Wilck. 192 & $8 / 05 / 143$ & 5 & 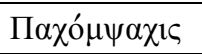 & $\mu \varepsilon \rho(1 \sigma \mu o ́ \varsigma) \beta^{5}$ & 12 dr. 3 ob. \\
\hline 16- $S B$ VI 9545 (20) & $14 / 07 / 143$ & $\varsigma$ & $\Pi \alpha \lambda \alpha ́ \chi \eta \mu 1 \varsigma^{6}$ & $\begin{array}{l}\lambda \alpha o \gamma \rho \alpha \operatorname{li}^{\alpha} \alpha \\
\varsigma \mathrm{L}\end{array}$ & 17 dr. 1 ob. \\
\hline
\end{tabular}

\footnotetext{
${ }^{4}$ Pour la lecture du nom, cf. note 13.

5 J. Shelton, «Ostraca from Elephantine in the Fitzwilliam Museum», ZPE 80, 1990, p. 233, envisage la résolution $\mu \varepsilon \rho(1 \sigma \mu \hat{\omega} v) \beta$ «pour deux merismoi». Pour le sens de $\mu \varepsilon \rho ı \sigma \mu o i ́$ sans autre détermination, cf. ibid., p. 232.

${ }^{6}$ Pour la lecture du nom, cf. note 13.
} 


\begin{tabular}{|c|c|c|c|c|c|}
\hline 17- O.Wilck. $194^{7}$ & $\begin{array}{l}31 / 10 / 143 \\
-------- \\
\text { 6/11/143 }\end{array}$ & $\zeta$ & 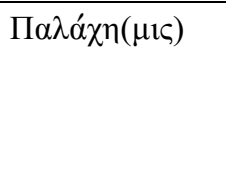 & 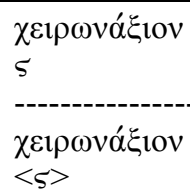 & $\begin{array}{l}12 \mathrm{dr} . \\
8 \mathrm{dr} .2 \text { ob. }\end{array}$ \\
\hline 18-O.Wilck. 193 & $141-143^{8}$ & [ ] & $\Pi[\alpha \chi o ́ \mu \psi \alpha \chi 1 \varsigma]^{9}$ & $\begin{array}{l}{\left[\chi \varepsilon 1 \rho \omega v \alpha^{\prime} \xi_{1-}\right.} \\
\text { ov] [x L] }\end{array}$ & 20 dr. 2 ob. \\
\hline 19- O.Bodl. II 2224 & $141-143^{10}$ & [ ] & $\Pi \alpha[\chi o ́ \mu \psi \alpha \chi 1 \varsigma]^{11}$ & $?$ & $?$ \\
\hline 20- P.Worp 46 & $141-143$ & [ ] & Пахó $\mu \psi \alpha \chi 1 \varsigma$ & 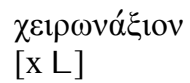 & $12 \mathrm{dr}$. \\
\hline
\end{tabular}

Hérakleidês et Isidôros sont représentés par le commis de bureau (ßon日óc) Pakhompsakhis, qui rédige la quittance. Ce dernier est connu dans cette fonction de 135/136 (O.Wilck. 167) jusqu'à 146 (O.Wilck. 207) ${ }^{12}$. Ce n'est pas le seul boêthos travaillant pour Hérakleidês et Isidôros: il y eut aussi Sarapiôn (O.Bodl. II 966; O.Leid. 182) et Palakhêmis (O.Bodl. II 1066 13 ; SB VI 9545 (20)) ${ }^{14}$.

L'apparition des fermiers ou épitérètes de la Porte sacrée de Syène ( $\mu 1 \sigma \theta \omega \tau \alpha \grave{/}$

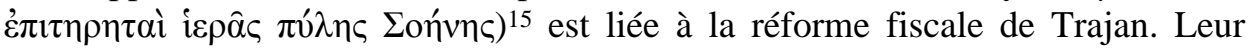
statut a fait débat: si Wilcken pensait que ces «fermiers» étaient en fait des liturges, encaissant les mêmes impôts que les praktores argyrikôn ${ }^{16}$, qui, à Syène/Éléphantine, se spécialisent dans la levée des impôts de répartition (merismoi), F. Reiter y voit pour sa part de vrais fermiers ${ }^{17}$ : en témoignent, selon lui, leurs gentilices romains qui tranchent sur les noms grecs des praktores qu'ils remplacent.

Hérakleidês et Isidôros encaissent ici deux impôts de nature différente: la $\lambda \alpha o-$

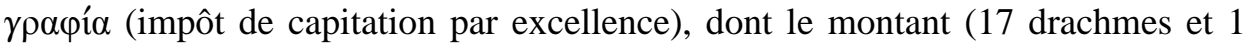

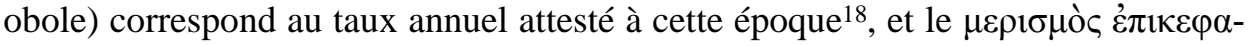

\footnotetext{
${ }^{7}$ Le reçu est émis par les épitérètes Tiberius Iulius Longinus et Ualerius Sôkratous accom-

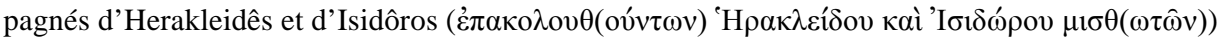

${ }^{8}$ L'éd. ne donne pas de date. O.Cair., p. 134: 139-143.

${ }^{9}$ En l'absence de photographie, on peut se demander si le reçu n'est pas de Palakhêmis.

10 Éd.: $139-141$

${ }^{11}$ Amin Benaissa a eu la gentillesse de contrôler l'original pour moi (ce dont je le remercie): il s'agit bien de l'écriture de Pakhompsakhis et non celle de Palakhêmis.

${ }^{12}$ Sur la carrière de ce personnage, cf. P.Worp 45, 2 n.

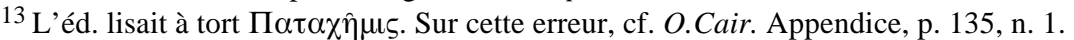

${ }^{14}$ Sur la carrière de Palakhêmis, cf. P.Worp, p. 287-289.

${ }^{15}$ Cf. S.L. Wallace, Taxation in Egypt from Augustus to Diocletian, Princeton 1938, p. 299.

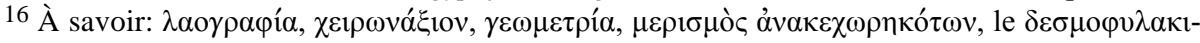

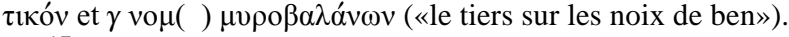

${ }^{17}$ F. Reiter, Die Nomarchen des Arsinoites. Ein Beitrag zum Steuerwesen im römischen Ägypten (Papyrologica Coloniensia, 31), Paderborn 2004, p. 123-127.

${ }^{18}$ Il était de 16 drachmes à Élephantine entre 12 et 92/93. Sur la capitation en général, cf. S.L. Wallace, Taxation in Egypt from Augustus to Diocletian, Princeton 1938, p. 116-134, et, à Éléphantine, cf. P.Brook. 34 intr. et P.Worp 45, 4 n.
} 


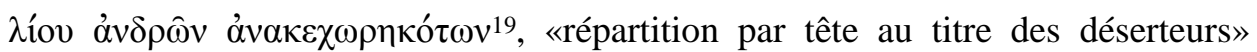
(contribution par répartition apparaissant sous Trajan et destinée à compenser le manque à gagner dû aux évadés fiscaux), d'un montant d'une drachme et 5 oboles $^{20}$ (cf. Tableau 2). Ces deux taxes se combinent souvent ${ }^{21}$; dans le dossier d'Herakleidês et d'Isidôros, on peut citer O.Wilck. 182 (25 juillet-23 août 141) et $183\left(1^{\text {er }}\right.$ oct. 141$)$.

C'est un fait bien connu que l'année au titre de laquelle est levé le $\mu \varepsilon \rho ı \mu$ ò

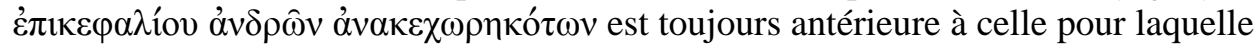
est due la $\lambda \alpha o \gamma \rho \alpha \varphi i^{\alpha}$, en général d'un an (ce qui crée un décalage d'un ou deux ans par rapport à la date d'émission du reçu). On constatera que, dans le cas présent, l'antériorité est très probablement de deux ans (si on restitue $\varepsilon$ ("̌́tovৎ) à la 1. 6 ${ }^{22}$ ); le décalage est en tout cas de trois ans avec la date d'émission du reçu. Cette anomalie trouve cependant des parallèles (P.Bingen 88, SB XX 15053 et O.Wilck. $182=\mathrm{n}^{\circ} 17,18$ et $19 \mathrm{du}$ Tableau 2), qui datent tous du début du règne d'Antonin.

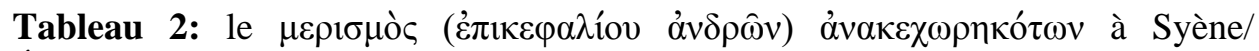
Éléphantine

\begin{tabular}{|c|c|c|c|c|c|}
\hline & $\begin{array}{l}\text { date } \\
\text { julienne }\end{array}$ & 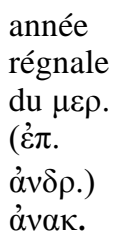 & $\begin{array}{l}\text { année régnale } \\
\text { de la } \lambda \alpha o \gamma \rho \alpha- \\
\text { pí } \alpha\end{array}$ & $\begin{array}{l}\text { année } \\
\text { régnale de } \\
\text { l'émission } \\
\text { du reçu }\end{array}$ & $\begin{array}{l}\text { montant } \\
\text { du } \mu \varepsilon \rho . \\
(\dot{\varepsilon} \pi . \dot{\alpha} v \delta \rho .) \\
\dot{\alpha} v \alpha \kappa .\end{array}$ \\
\hline $\begin{array}{l}\text { 1- } S B \text { VI } 9604 \\
\text { (20) }\end{array}$ & $21 / 08 / 115$ & $<>$ & - & in & 2 dr. 4 ob. \\
\hline 2-O.Wilck. 101 & $5 / 09 / 115$ & in & - & $1 \theta$ & 2 dr. 2 ob. \\
\hline 3-O.Wilck. $135^{23}$ & $3 / 08 / 126$ & $\varepsilon^{?}$ et $\varsigma^{?}$ & - & 1 & $2 \mathrm{dr} .1 \mathrm{ch}$. \\
\hline
\end{tabular}

\footnotetext{
${ }^{19}$ Le nom de cet impôt, toujours abrégé, a été mal résolu dans le passé: cf. J. Shelton, «Ostraca from Elephantine in the Fitzwilliam Museum», ZPE 80, 1990, p. 237-38. Il est typique de Syène-

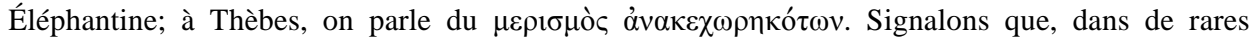
occurrences syènites, on trouve des formulations plus courtes: $\mu \varepsilon \rho 1 \sigma \mu \grave{c} \varsigma \dot{\alpha} v \delta \rho \omega \hat{\omega} v \dot{\alpha} v \alpha \kappa \varepsilon \chi \omega \rho \eta \kappa o ́ \tau \omega v$

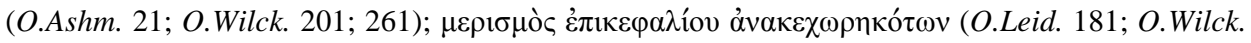

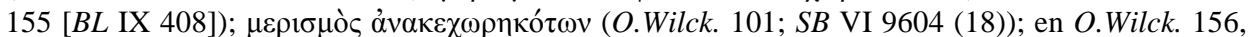
il faut réintroduire $\mu \varepsilon \rho 1 \sigma \mu \mathrm{o}(\hat{v})$ dans la lacune de la 1 . 6 (là où $B L$ IX 408 propose [áv $\delta(\rho \hat{\omega} v)$ $\dot{\alpha} v \alpha \kappa \varepsilon \chi \omega \rho(\eta \kappa o ́ \tau \omega v)(\ldots)]$. — Je garde la syntaxe proposée par Wallace selon laquelle $\varepsilon \dot{\pi} \iota \kappa \varepsilon \varphi \alpha \lambda$ íov est un substantif complément de nom de $\mu \varepsilon \rho ı \mu$ óc. On pourrait aussi penser que l'on a un adjectif

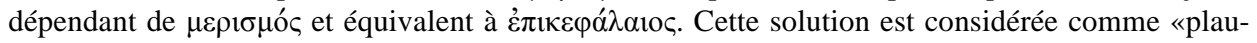
sible» par Shelton, l. c., p. 238, qui préfère cependant la construction de Wallace.

${ }^{20}$ Le taux varie: il est fixé chaque année en fonction des déficits du fisc.

21 Cf. J. Shelton, «Ostraca from Elephantine in the Fitzwilliam Museum», ZPE 80, 1990, p. 230, n. 6 .

22 Cf. note ad loc.

${ }^{23}$ Pour la provenance, cf. $B L$ II/1 49.
} 


\begin{tabular}{|c|c|c|c|c|c|}
\hline 4- O.Bodl. II 816 & $128 / 129$ & $\underline{1 \beta}$ & - & $1 \gamma$ & 1 dr. 2 ob. \\
\hline $\begin{array}{l}\text { 5- } S B \text { XXVI } \\
16497\end{array}$ & $129 / 130$ & $\gamma$ & {$[1] \delta$} & $1 \delta$ & $\begin{array}{l}1 \mathrm{dr} .[1 / 2 \\
\text { ob.] }\end{array}$ \\
\hline 6- O.Leid. 181 & $129 / 130$ & $1 \gamma$ & $1 \delta$ & $1 \delta$ & $1 \mathrm{dr} 1 / 2 \mathrm{ob}$ \\
\hline $\begin{array}{l}\text { 7- } S B \text { VI } 9604 \\
\text { (18) }\end{array}$ & $30 / 04 / 130$ & [ ] & $1 \delta$ & $1 \delta$ & 1 dr. $1 / 2$ ob. \\
\hline 8-O.Wilck. 152 & $130 / 131$ & $1 \delta$ & {$\left[1 \varepsilon^{24}\right]$} & $1 \varepsilon$ & $\begin{array}{l}1 \mathrm{dr} .1 \mathrm{ob} . \\
3 \mathrm{ch} .\end{array}$ \\
\hline 9- O.Wilck. 1272 & $\begin{array}{l}130- \\
132^{25}\end{array}$ & $1 \delta$ & {$\left[1 \varepsilon^{26}\right]$} & {$\left[1 \varepsilon\right.$ ou $\left.15^{27}\right]$} & $\begin{array}{l}1 \mathrm{dr} .1 \mathrm{ob} . \\
{\left[3 \text { ch. }^{28}\right]}\end{array}$ \\
\hline 10- O.Stras. I 284 & $1 / 06 / 131$ & $1 \delta$ & $1 \varepsilon$ & $1 \varepsilon$ & $\begin{array}{l}1 \mathrm{dr} .1 \mathrm{ob} . \\
2 \mathrm{ch}^{29}\end{array}$ \\
\hline $\begin{array}{l}\text { 11- SB VI } 9604 \\
\text { (19) }\end{array}$ & $22 / 11 / 131$ & $1 \delta$ & $1 \varepsilon$ & 15 & $\begin{array}{l}1 \mathrm{dr} .1 \text { ob. } \\
2 \mathrm{ch} .\end{array}$ \\
\hline 12- O.Wilck. 156 & $131 / 132$ & {$\left[1 \varepsilon^{30}\right]$} & 15 & 15 & $\begin{array}{l}1 \text { dr. [1 ob. } \\
\left.3 \text { ch. }{ }^{? 31}\right]\end{array}$ \\
\hline 13- O.Wilck. 155 & $131 / 132$ & {$\left[1 \delta^{32}\right]$} & $1 \varepsilon$ & 15 & $\begin{array}{l}1 \mathrm{dr} .1 \mathrm{ob} . \\
2 \mathrm{ch} .{ }^{33}\end{array}$ \\
\hline 14- O.Wilck. 154 & $14 / 08 / 132$ & $1 \varepsilon$ & 15 & 15 & $\begin{array}{l}1 \mathrm{dr} \text {. [1 ob. } \\
\left.3 \text { ch. }{ }^{? 34}\right]\end{array}$ \\
\hline 15- O.Wilck. 171 & $7 / 11 / 139$ & {$\left[\beta^{35}\right]$} & - & $\gamma$ & $\begin{array}{l}1 \text { dr. } 5 \text { ob. } \\
1 / 2\end{array}$ \\
\hline $\begin{array}{l}\text { 16- } S B \text { XIV } \\
11924\end{array}$ & $\begin{array}{l}28 / 12 / 139 \\
-26 / 01 / \\
140\end{array}$ & $\alpha$ & $\beta$ & $\gamma$ & [ ] \\
\hline 17- P.Bingen 88 & 139 & $\begin{array}{l}\text { In } \\
\text { d'Hadri- } \\
\text { en }\end{array}$ & - & $\alpha$ d'Antonin & $\begin{array}{l}1 \mathrm{dr} .4 \mathrm{ob} . \\
2 \mathrm{ch} .\end{array}$ \\
\hline 18- $S B$ XX 15053 & $139^{?}$ & $\begin{array}{l}\kappa \\
\text { d'Hadri- } \\
\text { en }\end{array}$ & к $\alpha$ d'Hadrien & $\begin{array}{l}{[\beta} \\
\text { d'Antonin] }\end{array}$ & [ ] \\
\hline 19-O.Wilck. 182 & $25 / 07-$ & $\beta$ & $\delta^{36}$ & $\delta$ & 2 dr. [ ] \\
\hline
\end{tabular}

${ }^{24} B L$ IX 408.

${ }^{25} B L$ IX 415

${ }^{26} B L$ IX 415

${ }^{27} B L$ IX 415

${ }^{28} B L \mathrm{II} / 1100$.

${ }^{29} B L$ IV 113.

${ }^{30} B L$ IX 408.

${ }^{31}$ Cette restitution est contestée par J. Shelton, ZPE 80, 1990, p. 230, n. 6.

${ }^{32} B L$ IX 408

${ }^{33} B L$ IX 408.

${ }^{34}$ Cette restitution est contestee par J. Shelton, ZPE 80, 1990, p. 230, n. 6 («as there is no adequate evidence on the rate charged at Syene in Hadrian's 15th year»).

${ }^{35}$ Le parallèle offert par $S B$ XIV $11924\left(n^{\circ} 16\right)$, très proche dans le temps, inciterait à restituer $\alpha$ plutôt que $\beta$. 


\begin{tabular}{|c|c|c|c|c|c|}
\hline & $23 / 08 / 141$ & & & & \\
\hline 20-O.Wilck. 183 & $1 / 10 / 141$ & $\gamma$ & $\delta$ & $\varepsilon$ & 1 dr. [ ] \\
\hline 21- O.Kauffmann & $\begin{array}{l}29 / 08- \\
27 / 09 / 142\end{array}$ & $\gamma$ & {$\left[\varepsilon^{?}\right]$} & 5 & 1 dr. 5 ob. \\
\hline 22- O.Wilck. 201 & $6 / 09 / 145$ & $\zeta$ & $\eta$ & $\theta$ & 1 dr. 4 ob. \\
\hline 23-O.Wilck. 261 & $\begin{array}{l}15 \text { ou } \\
15 / 10 / 161 \\
-168\end{array}$ & [ ] & [ ] & {$[$ ] } & $\begin{array}{l}{[1 \mathrm{dr} .] 2} \\
\text { ob. } 2 \text { ch. }\end{array}$ \\
\hline $\begin{array}{l}\text { 24- P.Eleph. } \\
\text { Wagner } 46\end{array}$ & $168 / 169$ & $\begin{array}{l}{[\zeta \text { ou }} \\
\left.n^{37}\right]\end{array}$ & - & $\theta$ & $\begin{array}{l}{[20 \text { dr. } 2 \text { ob. }} \\
2 \text { ch. }]^{38}\end{array}$ \\
\hline $\begin{array}{l}25-S B \text { XIV } \\
11925\end{array}$ & $12 / 10 / 171$ & [ ] & $1 \alpha$ & $1 \beta$ & [ ] \\
\hline 26- O.Bodl. II 460 & $7 / 09 / 178$ & {$[$ ] } & $m$ & $1 \theta$ & [ ] \\
\hline 27-O.Ashm. 21 & 194 & $\begin{array}{l}\alpha \\
\beta\end{array}$ & $\beta$ & [ ] & $\begin{array}{l}1 \mathrm{dr} .4 \mathrm{ob} . \\
2 \mathrm{dr} .1 \mathrm{ob} .\end{array}$ \\
\hline
\end{tabular}

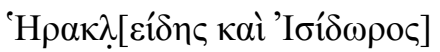

$\mu 1 \sigma \theta \omega \tau(\alpha i)[i \varepsilon \rho(\alpha \varsigma) \pi v ́ \lambda(n \varsigma) \Sigma o n ́ n \eta \varsigma]$

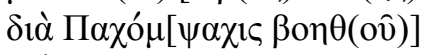

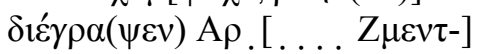

5

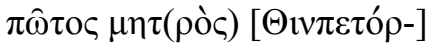

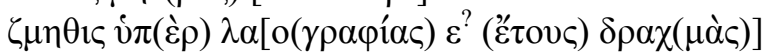

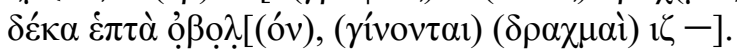

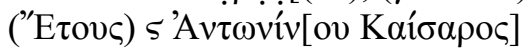

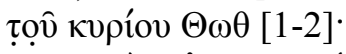

10

$\mu \varepsilon \rho ı \mu(\grave{o} v) \dot{\varepsilon} \pi ı \kappa(\varepsilon \varphi \alpha \lambda i ́ o v) \dot{\alpha} v \delta(\rho \hat{\omega} v)$



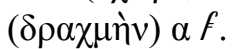

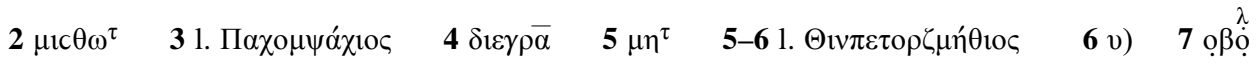
$\mathbf{8} \mathrm{L} \quad \mathbf{1 0} \mu \varepsilon \rho \stackrel{\mu}{\sigma} \varepsilon \pi \mathrm{\imath}^{\kappa} \alpha v^{\delta} \quad \mathbf{1 1} \alpha v \alpha^{\kappa}, \mathrm{L} \quad \mathbf{1 2}<$.

\footnotetext{
${ }^{36}$ Les années de la laographia et du merismos sont certaines (d'après l'image en ligne) malgré les doutes émis par J. Shelton, l. c., p. 230, n. 6 («WO has oddly $\lambda \alpha o \gamma \rho \alpha \varphi i ́ \alpha$ for year 4 and $\mu \varepsilon \rho 1 \sigma-$ $\mu$ óc for year 2 if the printed version is right»). Sur ce décalage de 2 ans, cf. ci-dessus.

37 Je propose de corriger ainsi la date; l'éditeur restitue $\theta$, qui est impossible étant donné le décalage d'au moins 1 à 2 ans entre l'année du merismos et celle de l'émission du reçu (cf. cidessus).

38 Je ne comprends pas bien ce qui motive cette restitution. Étant donné le caractère variable de cette contribution, il est plus prudent de ne rien restituer.
} 
«Hérakleidês [et Isidôros], fermiers [de la Porte sacrée de Syène], représentés par [le boêthos] Pakhompsakhis: ont été payés par Ar[---39 fils de Zment]pôs, ayant pour mère [Thinpetor]zmêthis, au titre de la capitation [de la $5^{\mathrm{e}}$ année] dix-sept [drachmes] et une obole. [Total: 17 dr. 1 ob.] An 6 d'Antonin César notre seigneur, le [x] Thôth.

Au titre de la quote-part par tête pour les déserteurs de la $3^{\mathrm{e}}$ année: $1 \mathrm{dr}$. et 5 ob.».

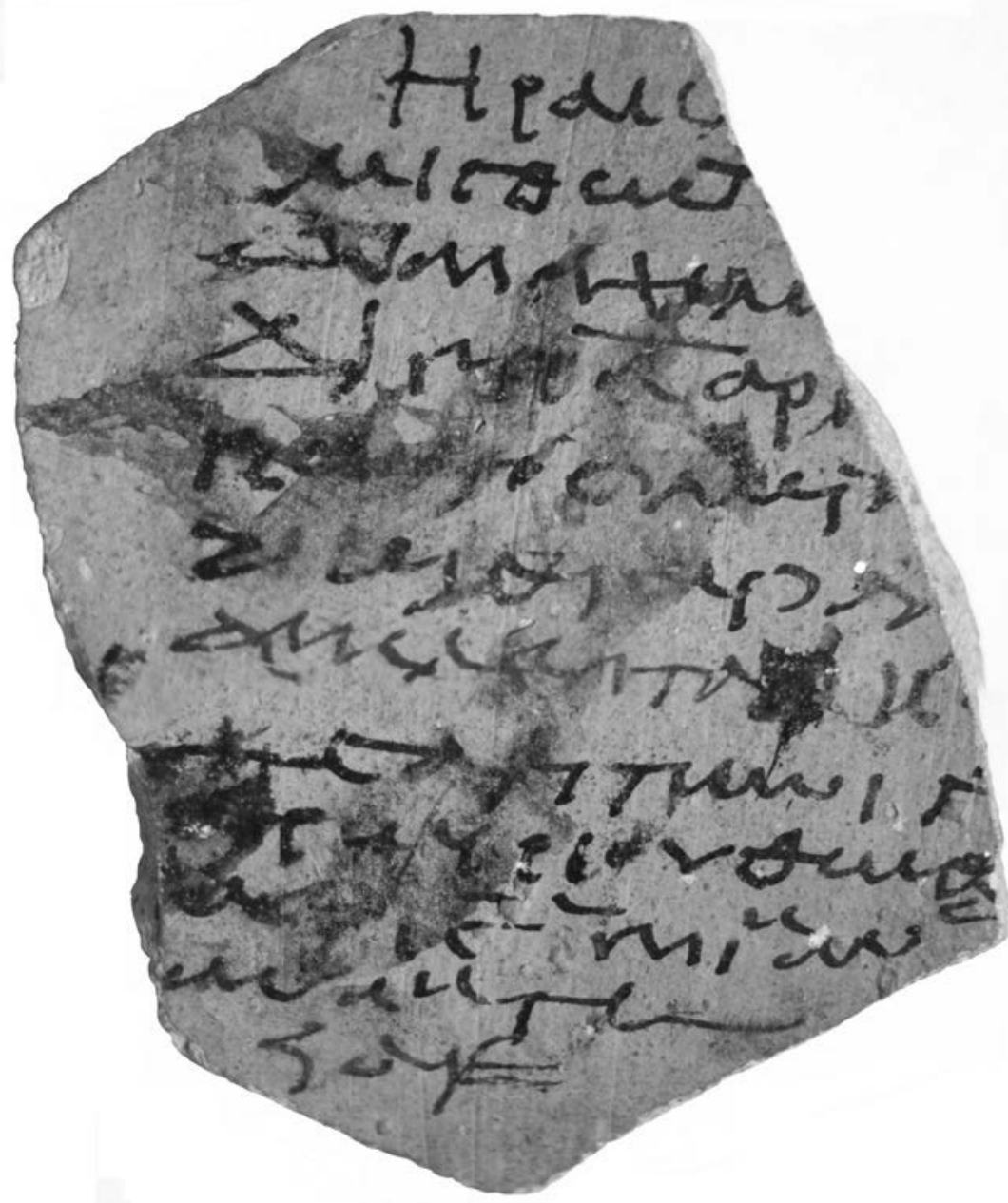

O. Kauffmann

${ }^{39}$ Ou Har[---]. 


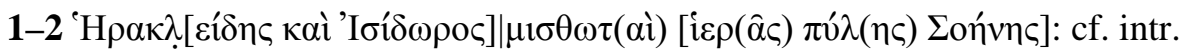

$2[i \varepsilon \rho(\hat{\alpha} \varsigma) \pi \hat{u} \lambda(\eta \varsigma) \Sigma o \eta ́ v \eta \varsigma]$ : on pourrait aussi restituer, dans la lacune, seulement $[\pi \hat{\lambda} \lambda(n \varsigma) \Sigma o \eta ́ v \eta \varsigma]$. L'expression courte est en effet attestée majoritairement sous le calame de Pakhompsakhis: O.Wilck. 181-183, 188, 189 (restitué), 191, 193, O.Eleph. Wagner 30, P.Köln II 115, P.Worp 46 (restitué) ${ }^{40}$. Mais, à moins que la ligne n'ait pas été entièrement écrite, la cassure oblique de l'ostracon oblige à restituer un texte plus long (dont la taille se situe entre celui de la 1.1 et celui de

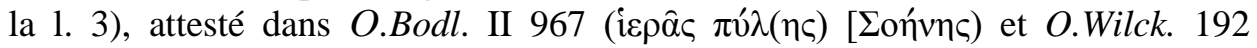
(i $\varepsilon \rho(\hat{\alpha} \varsigma) \pi v ́ \lambda(\eta \varsigma) \Sigma o \eta ́ v \eta \varsigma)$. Ces deux derniers parallèles montrent qu'on peut hésiter entre $i \varepsilon \rho(\hat{\alpha} \varsigma)$ et $i \varepsilon \rho \hat{\alpha} \varsigma$.


est typique de Syène-Éléphantine. Je restitue dans la lacune un nominatif comme dans le reste du dossier des reçus rédigés par Pakhompsakhis.

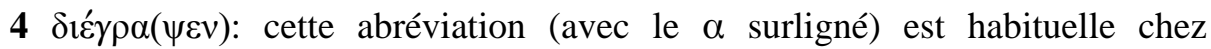
Pakhompsakhis comme en témoignent O.Wilck. 182, 183, 188, O.Eleph. Wagner 30, P.Köln II 115 (les éditeurs ne signalent pas la surligne au-dessus de l' $\alpha$, pourtant visible sur la photo), P.Worp 46.

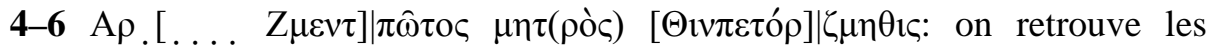

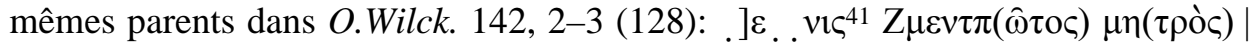
$\Theta \imath v \pi \varepsilon \tau \circ \rho \zeta \mu \eta ́(\theta ı \varsigma)$. Mais, d'après l'image, le nom du contribuable est différent; il doit d'agir d'un frère.

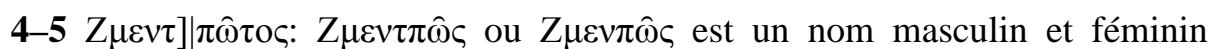
exclusivement de Syène-Éléphantine, correspondant à l'ég. Ns-mt(r)-p3-ç «Il appartient au $m t r$, le Grand». Le $m t(r)$ (qui remonte à la forme $m d w$ ) désigne l'enseigne sacrée du dieu Khnoum, vénérée à Éléphantine. Z $\mu \varepsilon v \tau \pi \omega \hat{\omega} \varsigma$ est une variation de $Z \mu \hat{\eta} \theta ı$, nom typique de cette région (ég. Ns-( $\left.p^{3}-\right) m t r$ «Il appartient au

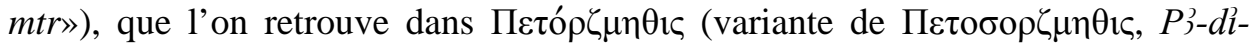
Wsir-ns-mtr «Celui qui est donné par Osorsmithis (= Osiris du $m t r) »)$ et dans le

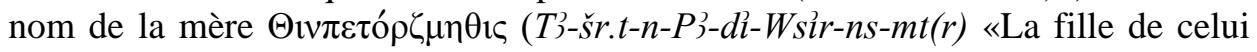
qui est donné par Osorsmithis»). Sur ces noms, cf. H. de Meulenaere, «L'enseigne sacrée du dieu Khnoum dans l'onomastique gréco-égyptienne», $C d E$ 75, 2000, p. $235-240$.

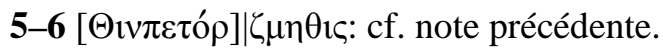

$6 \varepsilon^{?}$ ('̌́tovc): l'année est restituée d'après les parallèles proches dans le temps du nôtre, O.Wilck. 190 et 191 (cf. Tableau 1).



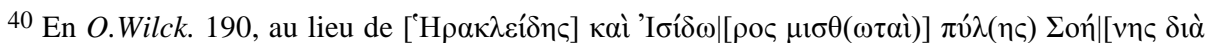

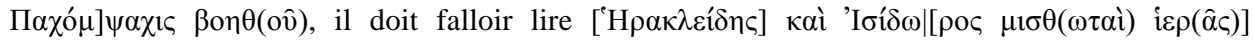

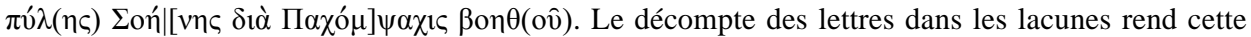
restitution préférable.

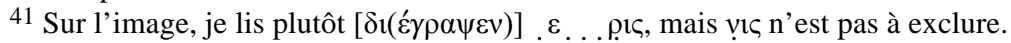


10-11 $\mu \varepsilon \rho ı \mu(\hat{o} v) \dot{\varepsilon} \pi \iota \kappa(\varepsilon \varphi \alpha \lambda i ́ o v) \dot{\alpha} v(\delta \rho \hat{\omega} v) \mid \dot{\alpha} v \alpha \kappa(\varepsilon \chi \omega \rho \eta \kappa o ́ \tau \omega v):$ cf. intr.

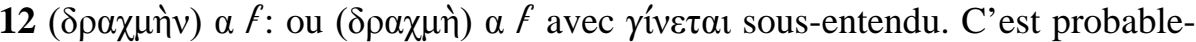
ment ce même montant qu'il faut restituer en $O$.Wilck. 183, 8 (éd: $\zeta \alpha{ }^{42}$ ).

${ }^{42}$ Et non $\alpha$ comme l'édite la $D D B D P$. Comme Wilcken ne fait pas de différence entre le point marquant une lettre illisible et le point de ponctuation (tous les deux à la même hauteur), le second doit être interprété comme un point de ponctuation (dont Wilcken fait précéder les dates). Cet exemple montre, s'il en est besoin, la nécessité d'employer, en papyrologie, le point sous la ligne... 